\title{
A Novel Mutation in PITX2 in a Patient with Axenfeld-Rieger Syndrome
}

\author{
Susan J. Hassed Shibo Li Weihong Xu Ashley C. Taylor \\ Section of Genetics, Department of Pediatrics, University of Oklahoma Health Sciences Center, \\ Oklahoma City, OK, USA
}

\section{Established Facts}

- Axenfeld-Rieger syndrome is a rare autosomal dominant condition.

- Mutations in the PITX2 gene have been identified in families with Axenfeld-Rieger syndrome.

- The mutation NM_153427.2:c.272G>A (p.Arg91Gln) in PITX2 has been reported in one individual, the patient reported herein, and classified as a variant of uncertain significance.

- Multiple lines of computational evidence predict this variant is probably damaging to the protein structure or function.

\section{Novel Insights}

- The mutation NM_153427.2:c.272G>A (p.Arg91Gln) in PITX2 segregates with the Axenfeld-Rieger phenotype in this family.

- The mutation is de novo in the affected mother of the patient. Maternal grandparents were tested and were negative.

- The variant can be classified as pathogenic according to the American College of Medical Genetics and Genomics 2015 rule Pathogenic(iii)(b), specifically rules PS2, PM2, PM5, PP1, and PP3.

\section{Key Words}

Axenfeld-Rieger syndrome · PITX2

\begin{abstract}
Axenfeld-Rieger syndrome is a rare autosomal dominant condition. Anomalies include anterior segment dysgenesis of the eye, dental anomalies, maxillary hypoplasia, periumbilical anomalies, and congenital heart defects. We report a patient with Peters anomaly, dysmorphic features, congenital heart defect, umbilical hernia, short stature, and develop-
\end{abstract}

mental delay. Diagnostic sequencing of 23 genes known to be causally related to the condition was performed on the patient, parents, and maternal grandparents. A variant of uncertain significance in PITX2 was identified. The mother had the same mutation and the father did not. The mother had decreased vision, congenitally missing teeth, and required jaw surgery as a child. Her asymptomatic parents elected to be tested and were negative for the mutation. The mutation, NM_153427.2:c.272G>A (p.Arg91Gln), is predicted to be damaging by PolyPhen-2 (score of 0.997 ), identified as a missense mutation with an allele frequency of $1.648 \mathrm{e}-05$ by the

\section{KARGER}

(c) 2017 S. Karger AG, Basel

E-Mail karger@karger.com

www.karger.com/msy
Susan J. Hassed, PhD

Department of Pediatrics, University of Oklahoma Health Sciences Center 1200 Children's Ave., Room 12100

Oklahoma City, OK 73104 (USA)

E-Mail Susan-Hassed@OUHSC.edu 
Fig. 1. Patient with Axenfeld-Rieger syndrome.
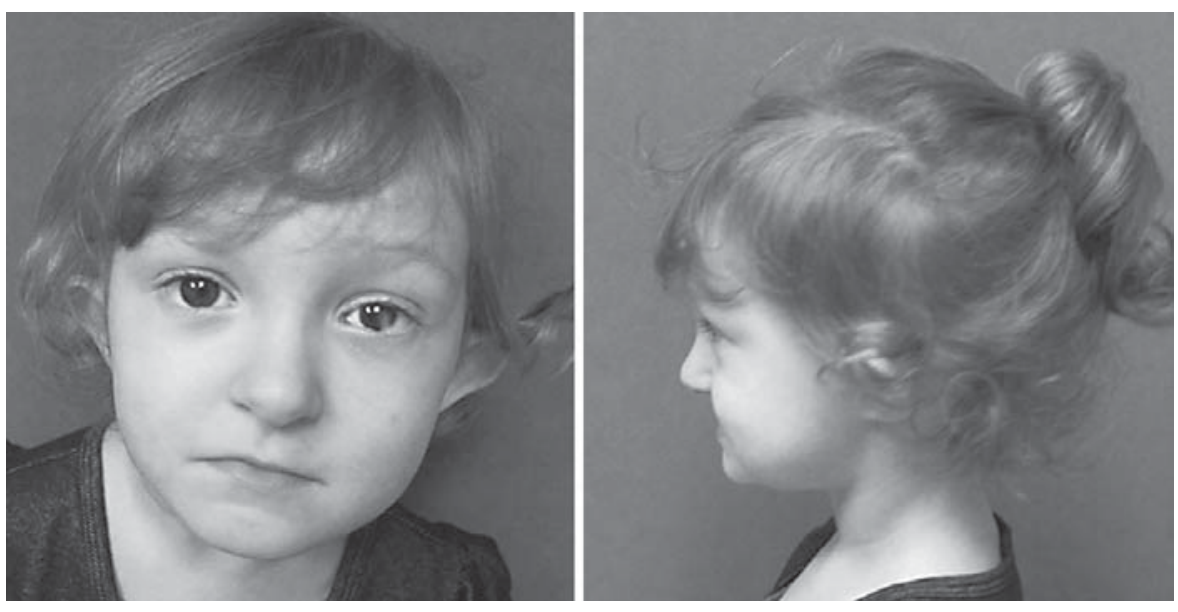

Exome Aggregation Consortium, and has been reported in ClinVar once, by the laboratory that analyzed our patient's sample. Due to the in silico predictions and the results of family studies, it is suggested that this variant can be classified as pathogenic according to the American College of Medical Genetics and Genomics 2015 rule Pathogenic(iii)(b), specifically rules PS2, PM2, PM5, PP1, and PP3.

(C) 2017 S. Karger AG, Basel

Axenfeld-Rieger syndrome, a rare autosomal dominant condition, encompasses a range of anomalies including anterior segment dysgenesis of the eye, dental anomalies, maxillary hypoplasia, periumbilical anomalies, and congenital heart defects [Alward, 2000; Tümer and Bach-Holm, 2009; Chang et al., 2012; Zhao et al., 2015; Sun et al., 2016]. Mutations in the PITX2 (pairedlike homeodomain transcription factor 2; OMIM 601542), and FOXC1 (forkhead box C1; OMIM 601090) genes have been identified in families with Axenfeld-Rieger syndrome. We report a patient with Peters anomaly, repaired cleft palate, dysmorphic facial features, congenital heart defect, mild umbilical hernia, short stature, mild developmental delay, and a familial variant of uncertain significance in PITX2.

\section{Patient and Methods}

Case Report

The girl, 3 years and 7 months old, was initially referred for evaluation of Peters anomaly, bilateral cleft palate, ventricular septal defect and patent foramen ovale as well as developmental delay (Fig. 1). On physical examination, height and weight were at the 2nd centile and head circumference 45th centile. Furthermore, left eye opacity and ptosis, cupped ears with overfolded helices, re- paired cleft palate, dentition appropriate for age, and small umbilical hernia were detected. The remainder of the physical examination was within normal limits. The mother reported that she had decreased vision greater on the right eye, congenitally missing teeth, and required surgery for repair of maxillary and mandibular hypoplasia. The father had no eye, dental, or related anomalies.

A skeletal survey for the patient was performed that revealed 13 pairs of ribs with corresponding vertebral bodies. Renal ultrasonogram was within normal limits. A blood sample was obtained for the sequencing of genes associated with anophthalmia/microphthalmia/anterior segment dysgenesis and revealed a change in PITX2 [NM_153427.2:c.272G>A (p.Arg91Gln)], classified as a variant of uncertain significance. The parents were tested and the mother was found to also have the mutation. Because of her past medical history of maxillary and mandibular hypoplasia as well as microgenia and the fact that neither of her parents have vision or dental anomalies, we tested her parents for the mutation and both were negative.

\section{Genetic Analysis}

Clinical molecular analysis was performed using an anophthalmia panel consisting of the following genes: B3GLCT, BCOR, BMP4, COL4A1, CYP1B1, FOXC1, FOXE3, FRAS1, FREM1, FREM2, GRIP1, HCCS, MFRP, NDP, OTX2, PAX6, PITX2, PITX3, SMOC1, SOX2, STRA6, VAX1, VSX2. A mutation was identified in the PITX2 gene associated with Axenfeld-Rieger syndrome. That mutation, NM_153427.2:c.272G $>$ A (p.Arg91Gln), is predicted to be damaging by PolyPhen-2 (score of 0.997), identified as a missense mutation with an allele frequency of $1.648 \mathrm{e}-05$ by the Exome Aggregation Consortium (http://exac.broadinstitute.org), and documented as a variant of uncertain significance in ClinVar by the laboratory that performed the analysis of our patient. Functional study for the same amino acid residue with Arg91Pro change shows this mutation leads to a dominant-negative factor that blocks the PITX2-activated promoters [Acunzo et al., 2011]. Multiple lines of computational evidence predict this variant is probably damaging to the protein structure or function.

The patient and her mother, who both have features of Axenfeld-Rieger syndrome, both carry the reported mutation in PITX2. The mother's parents neither have the same mutation nor are phenotypically affected with the condition. 


\section{Discussion}

Peters anomaly, an anterior segment dysgenesis disorder, may occur as an isolated single trait or in conjunction with other features. Disorders have been reported as sporadic, inherited as autosomal recessive conditions, or as autosomal dominant conditions. As specific genes have been identified as causally related to disorders of the anterior segment, the clinical spectrum is being clarified. Mutations in PITX2 have been reported to cause Axenfeld-Rieger syndrome Type 1 (OMIM 180500) [Tümer and Bach-Holm, 2009; Law et al., 2011; Chang et al., 2012; Li et al., 2014; Zhao et al., 2015; Seifi et al., 2016]. The patient reported herein has several features consistent with Axenfeld-Rieger syndrome: Peters anomaly, left eye opacity and ptosis, congenital heart defect, and small umbilical hernia. In addition, she had cupped ears with overfolded helices, bilateral cleft palate, and 13 ribs with corresponding vertebrae. The patient's mother had decreased vision in her right eye, a history of congenitally missing teeth, and surgery for repair of maxillary and mandibular hypoplasia. Neither of the mother's parents are phenotypically affected with the condition and nei- ther have the same mutation, suggesting a de novo event in the patient's mother. We suggest, based on in silico predictions and the family history reported: 2 affected individuals in 2 generations with unaffected grandparents proven not to have the mutation, that this variant can be classified as pathogenic according to the American College of Medical Genetics and Genomics 2015 rule Pathogenic(iii)(b), specifically rules PS2, PM2, PM5, PP1, and PP3.

\section{Statement of Ethics}

All laboratory testing in this family was performed to provide a clinical diagnosis and approved by the family via medical release. Clinical genetic testing of grandparents is covered by a protocol reviewed by the Institutional Review Board (No. 2866). Photographic permission was obtained and is on file.

\section{Disclosure Statement}

This clinical testing was not funded by a research grant. There are no conflicts of interest to report.

\section{References}

Acunzo J, Roche C, Defilles C, Thirion S, Quentien $\mathrm{MH}$, et al: Inactivation of PITX2 transcription factor induced apoptosis of gonadotroph tumoral cells. Endocrinology 152: 3884-3892 (2011).

Alward WLM: Axenfeld-Rieger syndrome in the age of molecular genetics. Am J Ophthalmol 130:107-115 (2000).

Chang TC, Summers CG, Schimmenti LA, Grajewski AL: Axenfeld-Rieger syndrome: new perspectives. Brit J Ophthalmol 96:318-322 (2012).
Law SK, Sami M, Piri N, Coleman AL, Caprioli J: Asymmetric phenotype of Axenfeld-Rieger anomaly and aniridia associated with a novel PITX2 mutation. Mol Vis 17:1231-1238 (2011).

Li X, Venugopalan SR, Cao H, Pinho FO, Paine ML, et al: A model for the molecular underpinnings of tooth defects in Axenfeld-Rieger syndrome. Hum Mol Genet 23:194-208 (2014).

Seifi M, Footz T, Taylor SAM, Elhady GM, Abdalla EM, Walter MA: Novel PITX2 gene mutations in patients with Axenfeld-Rieger syndrome. Acta Ophthalmol 94:e571-e579 (2016).
Sun YM, Wang J, Qiu XB, Yuan F, Xu YJ, et al: PITX2 loss-of-function mutation contributes to tetralogy of Fallot. Gene 577:258-264 (2016).

Tümer Z, Bach-Holm D: Axenfeld-Rieger syndrome and spectrum of PITX2 and FOXC1 mutations. Eur J Hum Genet 17:1527-1539 (2009).

Zhao CM, Peng LY, Li L, Liu XY, Wang J, et al: PITX2 loss-of-function mutation contributes to congenital endocardial cushion defect and Axenfeld-Rieger syndrome. PLoS One 10: e0124409 (2015) 